\title{
Ursolic acid prevents augmented peripheral inflammation and inflammatory hyperalgesia in high-fat diet-induced obese rats by restoring downregulated spinal PPAR $\alpha$
}

\author{
YANAN ZHANG ${ }^{1}$, CHENGWEI SONG $^{1}$, HAIOU LI ${ }^{1}$, JINGDONG HOU ${ }^{1}$ and DONGLIANG LI ${ }^{2}$ \\ ${ }^{1}$ Department of Anesthesiology, Jining First People's Hospital, Jining, Shandong 272011; \\ ${ }^{2}$ Department of Anesthesiology, Qilu Hospital, Shandong University, Jinan, Shandong 250012, P.R. China
}

Received June 7, 2015; Accepted April 2, 2016

DOI: $10.3892 / \mathrm{mmr} .2016 .5172$

\begin{abstract}
Obesity is a risk factor for several pain syndromes and is associated with increased pain sensitivity. Evidence suggests that obesity causes the downregulation of peroxisome proliferator-activated receptor (PPAR) $\alpha$ in the spinal cord, contributing to augmented peripheral edema and inflammatory hyperalgesia. Ursolic acid (UA), a natural pentacyclic triterpenoid carboxylic acid, has been shown to upregulate PPAR $\alpha$ in the peripheral tissues of obese animals. The present study hypothesized that UA prevents augmented peripheral inflammation and inflammatory hyperalgesia in obesity by restoring downregulated spinal PPAR $\alpha$. The present study demonstrated that Sprague-Dawley rats fed a high-fat diet (HFD) for 12 weeks developed obesity and metabolic disorder. Following carrageenan injection, the HFD rats exhibited increased thermal hyperalgesia and paw edema, compared with the rats fed a low-fat diet. Molecular investigations revealed that the HFD rats exhibited decreased PPAR $\alpha$ activity, and exaggerated expression of inflammatory mediators and nuclear factor-kB activity in the spinal cord in response to carrageenan. Oral administration of UA ameliorated obesity and metabolic disorder, and prevented increased thermal hyperalgesia and paw edema in the HFD rats. Additionally, UA normalized PPAR $\alpha$ activity and inhibited the exaggerated spinal cord inflammatory response to carrageenan. Although the knockdown of spinal PPAR $\alpha$ with small interfering RNA following the administration of UA did not alter obesity or metabolic parameters, it eradicated the beneficial effects of UA on thermal hyperalgesia and paw edema, and reversed the spinal cord inflammatory response. These results suggested that the systemic administration of UA
\end{abstract}

Correspondence to: Dr Jingdong Hou, Department of Anesthesiology, Jining First People's Hospital, 6 Jiankang Road, Jining, Shandong 272011, P.R. China

E-mail: hou-jd@hotmail.com

Key words: ursolic acid, obesity, peroxisome proliferator-activated receptor- $\alpha$, pain, inflammation inhibited the exaggerated spinal cord inflammatory response to peripheral inflammatory stimulation in HFD-induced obesity by restoring downregulated spinal PPAR $\alpha$, preventing peripheral inflammation and inflammatory hyperalgesia. UA may be a potential therapeutic option for the prevention of increased inflammatory pain in obese patients.

\section{Introduction}

The rise in global obesity rates over the last three decades has been substantial and widespread, becoming a major public health epidemic in developed and developing countries (1-3). Obesity is also well-recognized as a risk factor associated with multiple pain syndromes, including lower back pain, tension-type headaches and migraines, fibromyalgia, abdominal pain and chronic widespread pain (4-7). These pain conditions are not solely due to mechanical stress on the joints due to increased load, other factors have been suggested to be involved in the progression of chronic pain in obesity.

Evidence from experimental studies has demonstrated that obesity is associated with increased peripheral inflammation and pain sensitivity in response to inflammatory stimulation (8-10). The spinal cord is the predominant relay station in the neural communication between inflamed areas and the central nervous system (CNS) (11). Obesity has been shown to induce the downregulation of spinal peroxisome proliferator-activated receptor (PPAR) $\alpha$, which sensitizes peripheral inflammatory stimulation by increasing the inflammatory response in the spinal cord, contributing to augmented peripheral inflammation and inflammatory hyperalgesia (12). Thus, upregulating PPAR $\alpha$ in the spinal cord may offer a novel therapeutic strategy for preventing augmented peripheral inflammation and inflammatory hyperalgesia in obesity.

Ursolic acid (UA), a natural pentacyclic triterpenoid carboxylic acid, is the major component of several traditional medicine herbs and has multiple biological activities, including the regulation of lipid and glucose metabolism $(13,14)$, in addition to anti-oxidative, antimutagenic, anticarcinogenic, antimicrobial and anti-atherosclerotic effects (15-17). UA also exhibits analgesic and anti-inflammatory activities in normal animals $(18,19)$, however, the molecular mechanism, and whether UA has beneficial effects on inflammatory 
hyperalgesia in obesity remain to be elucidated. Previous studies have demonstrated that UA increases the expression and activity of PPAR $\alpha$ in the liver of obese animals $(13,14)$, and UA has been shown to cross the blood brain barrier $(20,21)$. The present study was performed to test the hypothesis that UA prevents increased peripheral inflammation and inflammatory hyperalgesia in obesity by restoring downregulated spinal PPAR $\alpha$. For this purpose, a diet-induced rat model of obesity was used, which has been considered to be the most relevant model with regard to human obesity (22).

\section{Materials and methods}

Animals. Adult male Sprague-Dawley rats weighing $85 \pm 3 \mathrm{~g}$ were purchased from the Beijing Laboratory Animal Research Center (Beijing, China). They were housed in a room maintained at $23-25^{\circ} \mathrm{C}$ on a 12 -h-light/dark cycle, and were provided with rat chow and water ad libitum. The study was approved by the ethics committee of Shandong University (Shandong, China), and all animal experiments were performed in accordance with the guidelines of the Institutional Animal Care and Use Committee of Shandong University.

Experimental protocol. Following 1 week of adaptation to their housing environment, the rats were randomly divided into the following groups ( $n=18$ in each group): i) High-fat diet (HFD) control group, comprising HFD-fed rats without treatment; ii) HFD+UA (Sigma-Aldrich, St. Louis, MO, USA), comprising HFD-fed rats treated with UA; iii) HFD+UA+PPAR $\alpha$ small interfering (si)RNA, comprising HFD-fed rats treated with UA and PPAR $\alpha$ siRNA; iv) HFD+UA+scrambled siRNA, comprising HFD-fed rats treated with UA and scrambled siRNA; v) low-fat diet (LFD), comprising LFD-fed rats without treatment.

The rats, which were assigned to the HFD or LFD groups were fed a HFD $(45 \%$ kcal as fat; Research Diets, New Brunswick, NJ, USA) or a LFD (10\% kcal as fat) for 12 weeks, respectively. UA ( $250 \mathrm{mg} / \mathrm{kg} /$ day) was orally administered for 8 weeks, starting from week 4, when the HFD-fed rats had become significantly heavier, compared with the LFD-fed rats (12). The dose of UA was based on a previous study (14). PPAR $\alpha$ siRNA $(2 \mu \mathrm{g} / 10 \mu \mathrm{l})$ or scrambled siRNA was intrathecally injected at 10 weeks, as previously described (12). Body weights were measured weekly. At the end of the experiments, 12 weeks post-HFD or LFD feeding), six rats from each group were sacrificed by decapitation using a guillotine (Kent Scientific Corporation, Torrington, CT, USA) and 5-ml blood samples were collected for the measurement of metabolic parameters, and the spinal cord was removed for assessment of the expression levels of PPAR $\alpha$ and inflammatory mediators, and the activity of nuclear factor $(\mathrm{NF})-\kappa \mathrm{B}$ at baseline. Other rats ( $\mathrm{n}=12$ rats in each group) received a subcutaneous injection of 3\% carrageenan (50 $\mu \mathrm{l}$; Sigma-Aldrich) into the mid-plantar region of the right hindpaw, as previously described (12). The thermal and mechanical responses of the right hindpaw, and the paw volumes of the right and left hindpaws were then measured at baseline, and at 2, 4, 6, 8 and $24 \mathrm{~h}$ following carrageenan injection. At $6 \mathrm{~h}$ post-carrageenan injection, six rats from each group were sacrificed by decapitation using a guillotine to assess the expression levels of PPAR $\alpha$ and inflammatory mediators, and the activity of NF- $\kappa \mathrm{B}$ in the spinal cord in response to carrageenan injection. The remaining rats were sacrificed $24 \mathrm{~h}$ after carrageenan injection by decapitation using a guillotine.

Intrathecal injection of siRNA. PPAR $\alpha$ siRNA (Shanghai GenePharma Co., Ltd., Shanghai, China) was constructed using IMG-800 vector (pSuppressorNeo; Imgenex, San Diego, CA, USA), as previously described (12). The siRNA target sequence used for the construction of the PPAR $\alpha$ plasmid vector was: 5'-TCGAGTGTGATCGAAGCTGCAAGATGG AATTCGATCTTGCAGCTTCGATCACACTTTTT-3'. The PPAR $\alpha$ siRNA or scrambled siRNA (Shanghai GenePharma) was suspended in serum-free media and intrathecally injected in a volume of $10 \mu \mathrm{l}$ using a $25-\mu 1$ Hamilton microsyringe (Hamilton Co., Reno, NV, USA) fitted with a 27-gauge needle. The needle was inserted into the subarachnoid space through the intervertebral foramen. The correct placement of the needle was verified by a tail or foot flick response.

Hindpaw withdrawal responses to thermal and mechanical stimulation. Prior to the assessment of response to thermal and mechanical stimulation, the animals underwent acclimatization to the assessment environment for 1 week by placing them in the thermal and mechanical assessment boxes for 30-60 min twice daily. The hindpaw withdrawal responses to thermal stimulation were assessed using a plantar test instrument (\#7370; Ugo Basile; Comerio, VA, Italy), as previously described (12). The thermal stimulation was performed using an infrared beam, which was directed onto the plantar surface of the hindpaw, and the latency to paw withdrawal was recorded. At each time point $(0,2,4,6,8$ and $24 \mathrm{~h}$ after the carrageenan injection), three measurements were performed and the average value was calculated. A cut-off value of $25 \mathrm{sec}$ was applied in the absence of a response to prevent tissue damage. The hindpaw withdrawal responses to mechanical stimulation were assessed using a dynamic plantar esthesiometer (\# 37400; Ugo Basile), which consisted of a filament, which touched the plantar surface of the hindpaw and began to exert an upwards force until the paw was withdrawn. A cut-off of $50 \mathrm{~g}$ was applied to prevent tissue damage.

Assessment of paw edema. Paw edema was measured using a plethysmometer (\#7140; Ugo Basile), as described previously (12). The paw volume was determined based on the displacement of water when the paw was submerged into the water. The volumes of the left and the right paws were measured, and the changes in paw volume were calculated as the percentage difference in volume between the ipsilateral and contralateral paw.

Western blot analysis. Nuclear and cytoplasmic fractions were extracted from the spinal cord using a Nuclear Extract kit (cat. no. 40010; Active Motif, Carlsbad, CA, USA). For the assessment of inflammatory mediators, protein was isolated from the spinal cord using cell lysis buffer $(20 \mathrm{mM}$ Tris- $\mathrm{HCl}, 150 \mathrm{mM}$ $\mathrm{NaCl}, 1 \mathrm{mM}$ EDTA-Na $2,1 \mathrm{mM}$ EGTA, $1 \%$ Triton X-100, $2.5 \mathrm{mM}$ sodium pyrophosphate, $1 \mathrm{mM} \beta$-glycerophosphate, $1 \mathrm{mM} \mathrm{Na} \mathrm{VO}_{4}$ and $1 \mu \mathrm{g} / \mathrm{ml}$ leupeptin; Cell Signaling Technology, Inc., Beverly, MA, USA). The protein concen- 
A

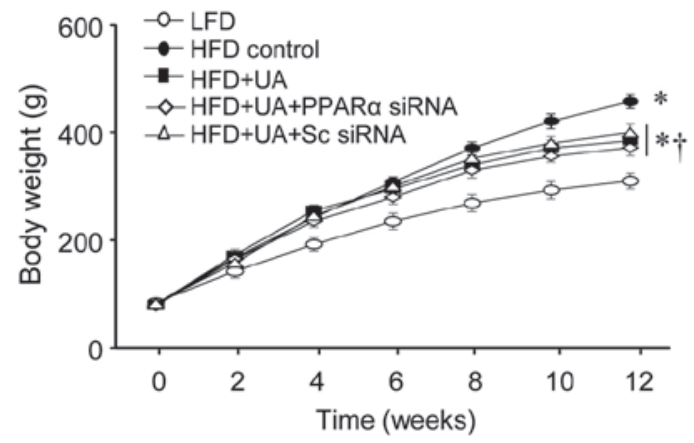

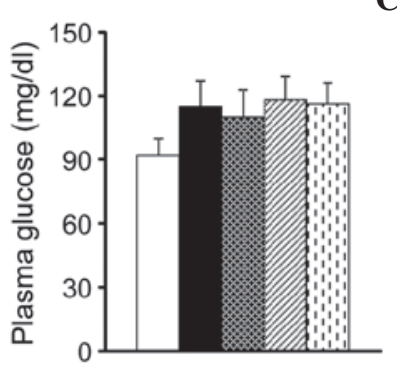

C

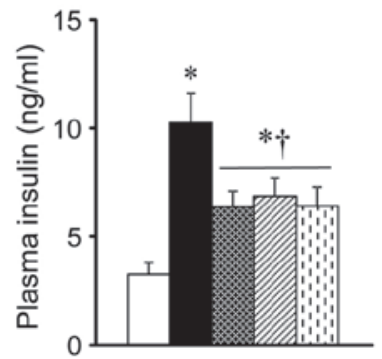

D

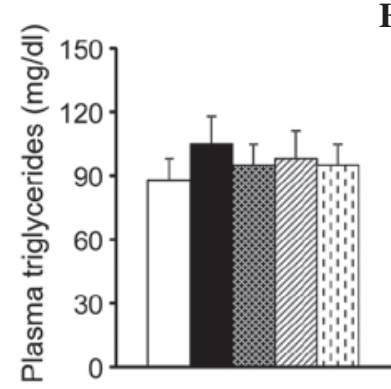

$\mathbf{E}$

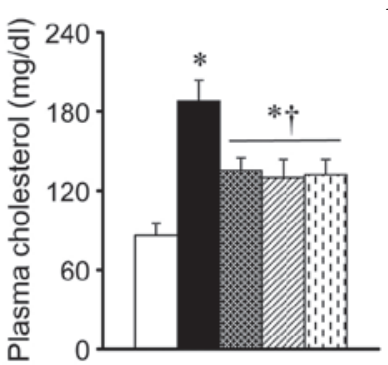

$\mathbf{F}$

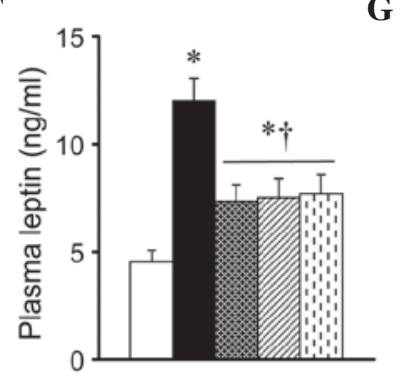

G

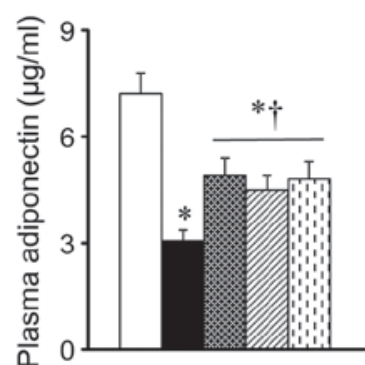

$\square$ LFD

HFD control

HFD+UA

WFD+UA+PPAR

[.] HFD+UA+Sc SiRNA

Figure 1. Effects of UA on body weight gain and metabolic parameters in HFD-induced obese rats. UA was orally administered for 8 weeks, starting 4 weeks following provision of the respective diets. PPAR $\alpha$ siRNA or Sc siRNA was intrathecally injected at week 10. (A) Body weights and plasma levels of (B) glucose, $(C)$ insulin, (D) triglycerides, $(\mathrm{E})$ cholesterol, $(\mathrm{F})$ leptin and $(\mathrm{G})$ adiponectin were examined. Data are presented as the mean \pm standard error of the mean. ${ }^{*} \mathrm{P}<0.05$ vs. LFD; ${ }^{\dagger} \mathrm{P}<0.05$, vs. HFD control. UA, ursolic acid; HFD, high-fat diet; LFD, low-fat diet; Sc, scrambled; siRNA, small interfering RNA; PPAR $\alpha$, peroxisome proliferator-activated receptor $\alpha$.

tration was determined by a Bradford assay (Bio-Rad Labs Informatics Division, Philadelphia, PA, USA). Briefly, dye reagent was prepared (dilution, 1 part dye reagent concentrate with 4 part double-distilled water). The bovine serum albumin (BSA) protein standard was prepared by making serial dilutions from a stock solution. $10 \mu \mathrm{l}$ BSA protein standards and samples were added and a blank of double-distilled water was used. After incubation for $5 \mathrm{~min}$ at room temperature, the absorbance was measured at $595 \mathrm{~nm}$ on a microplate reader (EL311S; Bio-Tek Instruments, Winooski, VT, USA). The optical density readings were taken and a standard curve was generated. The concentrations of samples were obtained based on the standard curve.

Nuclear protein levels of PPAR $\alpha$ and NF- $\kappa \mathrm{B}$ p 65 , protein levels of interleukin (IL)- $1 \beta$, tumor necrosis factor (TNF)- $\alpha$, cyclooxygenase (COX)-2 and inducible nitric oxide synthase (iNOS), and cytoplasmic protein levels of inhibitor of $\mathrm{NF}-\kappa \mathrm{B} \alpha(\mathrm{I} \kappa \mathrm{B} \alpha)$, were detected using western blot analysis. Briefly, $20 \mu \mathrm{g}$ protein was loaded onto $10 \%$ SDS-polyacrylamide gel (Bio-Rad Labs Informatics Division). Following electrophoresis ( $200 \mathrm{~V}$ for $35 \mathrm{~min}$ ), proteins were transferred to a polyvinylidene fluoride membrane (Bio-Rad Laboratories, Inc., Hercules, CA, USA). The membrane was blocked with Tris-buffered saline and Tween-20 (Bio-Rad Labs Informatics Division) with 5\% skimmed milk, and incubated overnight at $4^{\circ} \mathrm{C}$ with the following primary antibodies (all purchased from Santa Cruz Biotechnology, Inc., Dallas, TX, USA): Rabbit polyclonal anti-human IL-1 $\beta$ (cat. no. sc-7884; 1:200); goat polyclonal anti-human TNF- $\alpha$ (cat. no. sc-1350; 1:400); goat polyclonal anti-human COX-2 (cat. no. sc-23983; 1:400); rabbit polyclonal anti-mouse iNOS (cat. no. sc-650; 1:200); rabbit polyclonal anti-human NF- $\mathrm{B}$ p65 (cat. no. sc-109; 1:200); rabbit polyclonal anti-human $\mathrm{I} \kappa \mathrm{B} \alpha$ (cat. no. sc-847; 1:300); rabbit polyclonal anti-human PPAR- $\alpha$ (cat. no. sc-9000; 1:200); and goat polyclonal anti-human $\beta$-actin (cat. no. sc-1615; 1:1,000). After washing three times, the membrane was incubated for $1 \mathrm{~h}$ at room temperature with the following secondary antibodies (all purchased from Santa Cruz Biotechnology, Inc.): Goat anti-rabbit horseradish peroxidase conjugated secondary antibody (cat. no. sc-2030; 1:5,000) or donkey anti-goat horseradish peroxidase conjugated secondary antibody (cat. no. sc-2020; 1:5,000). After washing three times, the signals of the detected proteins were visualized using an enhanced chemiluminescence reaction system (EMD Millipore, Billerica, MA, USA). The density of the bands was analyzed using National Institutes of Health Image J software (version 1.48; NIH, Bethesda, MD, USA) and all data were normalized to $\beta$-actin.

$P P A R \alpha$ and $N F-\kappa B$ p 65 activity. The DNA binding activity of PPAR $\alpha$ and NF- $\mathrm{NB}$ p65 in the spinal nuclear extraction were measured using Transcription Factor Assay kits (cat. no. ab13112; Abcam, Cambridge, MA, USA and Active Motif), according to the manufacturer's protocols.

Biochemical measurements. Whole blood samples were centrifuged at $2,100 \mathrm{x}$ g for $15 \mathrm{~min}$ at $4^{\circ} \mathrm{C}$ and the plasma from each blood sample was collected. The plasma levels of glucose, total cholesterol and triglyceride were measured using a Roche Hitachi 911 Chemistry Analyzer (Hitachi Inc., 
A

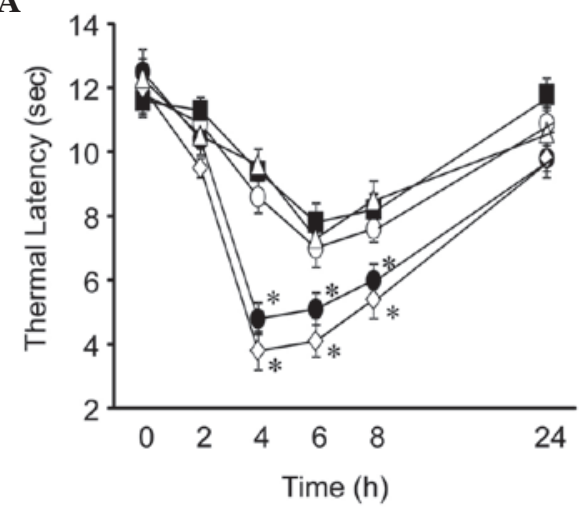

C

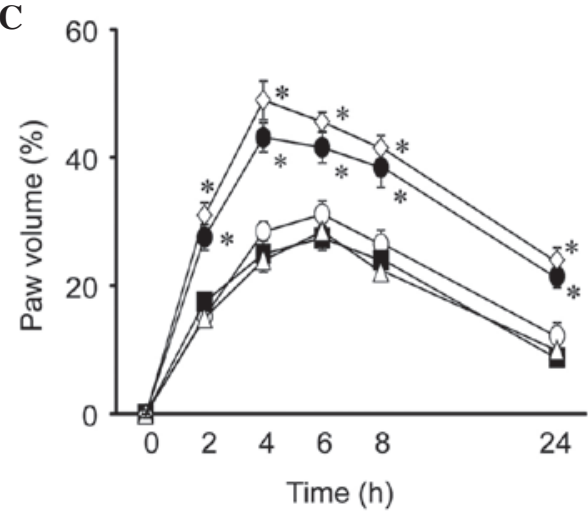

B

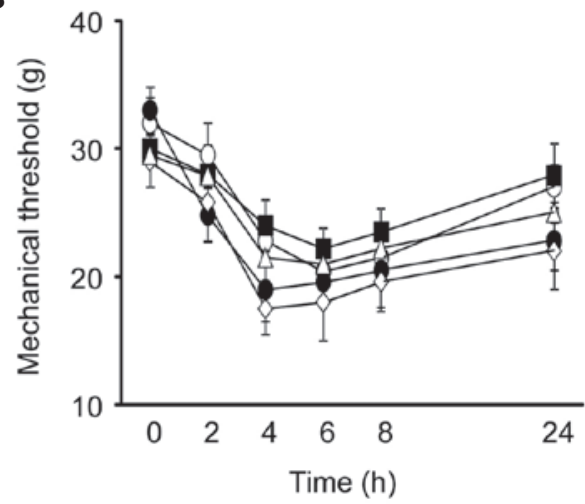

Figure 2. Effects of UA on thermal and mechanical hyperalgesia and paw edema. Effects of UA on (A) paw withdrawal latency, (B) paw withdrawal threshold and $(\mathrm{C})$ paw volume at baseline, and 2, 4, 6, 8 and $24 \mathrm{~h}$ post-carrageenan injection. Data are presented as the mean \pm standard error of the mean. ${ }^{*} \mathrm{P}<0.05, \mathrm{vs}$. LFD. UA, ursolic acid; HFD, high-fat diet; LFD, low-fat diet; Sc, scrambled; siRNA, small interfering RNA; PPAR $\alpha$, peroxisome proliferator-activated receptor $\alpha$.

Pleasanton, CA, USA). The plasma levels of insulin, leptin and adiponectin were analyzed using Invitrogen rat ELISA kits (cat. nos. ERINS, KRC2281 and KRP0041, respectively; Thermo Fisher Scientific, Inc., Waltham, MA, USA) according to the manufacturer's protocols.

Statistical analysis. GraphPad Prism 6 (GraphPad Software, Inc., La Jolla, CA, USA) was used for data analysis. Data are presented as the mean \pm standard error of the mean. The differences between groups were analyzed by two-way analysis of variance, followed by Bonferroni post-hoc tests for multiple comparisons. $\mathrm{P}<0.05$ was considered to indicate a statistically significant difference.

\section{Results}

Effects of UA on body weight and metabolic parameters. As shown in Fig. 1, body weights were significantly higher throughout the investigation in all HFD groups, compared with those in the LFD group, beginning at week 4. No differences in body weight were observed among the four HFD groups at 4 weeks. Compared with the HFD control rats, the HFD+UA rats exhibited significantly reduced body weight gain at 12 weeks. The intrathecal injections of PPAR $\alpha$ siRNA or scrambled siRNA had no effects on body weight gain in the HFD+UA rats.

At 12 weeks, the plasma levels of insulin, leptin and cholesterol were higher, and the plasma levels of adiponectin were lower in the HFD control rats, compared with those in the LFD rats. Compared with the HFD control rats, the HFD+UA rats exhibited significant improvements in all the above parameters. The intrathecal injections of PPAR $\alpha$ siRNA and scrambled siRNA did not alter these metabolic parameters in the HFD+UA rats. No differences in the plasma levels of glucose or triglycerides were observed between the experimental groups.

Effects of UA on thermal and mechanical hyperalgesia and paw edema. No differences were observed in thermal hyperalgesia, mechanical allodynia or paw edema between the experimental groups at baseline (prior to carrageenan injection), as shown in Fig. 2. Following carrageenan injection, all groups of rats exhibited significant thermal hyperalgesia, mechanical allodynia and paw edema in the injected paw. The maximum decreases in thermal response latency and mechanical response threshold, and maximum increase in paw volume, were observed in the carrageenan-injected paw at $6 \mathrm{~h}$ in the LFD rats, but at $4 \mathrm{~h}$ in the HFD control rats. Compared with the LFD rats, the HFD control rats had more pronounced thermal hyperalgesia between 4 and $8 \mathrm{~h}$ following carrageenan injection (Fig. 2A). Compared with the HFD control rats, the HFD+UA rats exhibited significantly attenuated thermal hyperalgesia. Of note, the intrathecal injection of PPAR $\alpha$ siRNA, but not of scrambled siRNA, completely eliminated the beneficial effect of UA on thermal hyperalgesia in the $\mathrm{HFD}+\mathrm{UA}$ rats in response to carrageenan injection. No significant differences were observed in the mechanical response thresholds between the experimental groups throughout the experimental period (Fig. 2B). Compared with the LFD rats, 
A

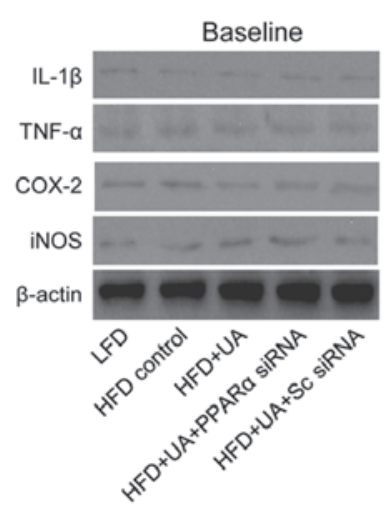

B

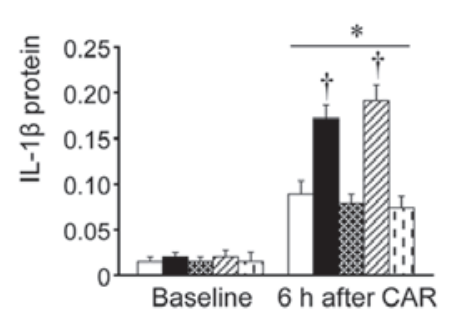

D

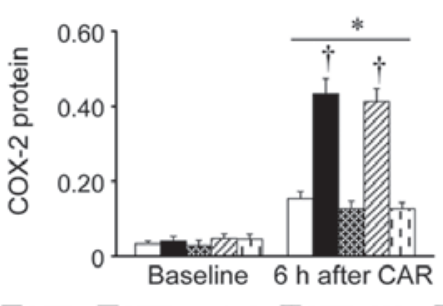

C

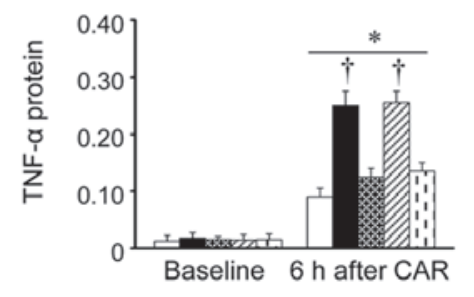

E

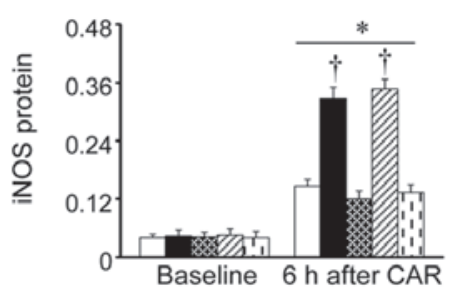

$\square$ LFD HFD control $\mathrm{xHFD+UA} \square \mathrm{HFD+UA+PPAR} \alpha$ siRNA $\square \mathrm{HFD+UA+Sc} \mathrm{siRNA}$

Figure 3. Effects of UA on expression levels of inflammatory mediators in the spinal cord at baseline and $6 \mathrm{~h}$ following CAR injection. (A) Representative western blots from each group are shown. The expression levels of (B) IL-1 $\beta,(\mathrm{C})$ TNF- $\alpha$; (D) COX-2 and (E) iNOS were quantified. Data were corrected by $\beta$-actin. Data are presented as the mean \pm standard error of the mean. ${ }^{*} \mathrm{P}<0.05$, vs. respective baseline; ${ }^{\dagger} \mathrm{P}<0.05$, vs. LFD under the same condition. UA, ursolic acid; HFD, high-fat diet; LFD, low-fat diet; CAR, carrageenan; Sc, scrambled; siRNA, small interfering RNA; IL-1 $\beta$, interleukin-1 $\beta$; TNF- $\alpha$, tumor necrosis factor- $\alpha$; COX-2, cyclooxygenase-2; iNOS, inducible nitric oxide synthase; PPAR $\alpha$, peroxisome proliferator-activated receptor $\alpha$.

A

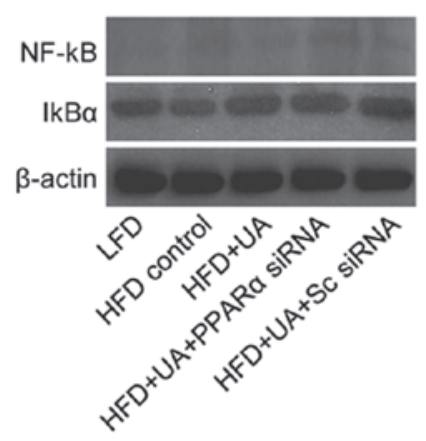

C

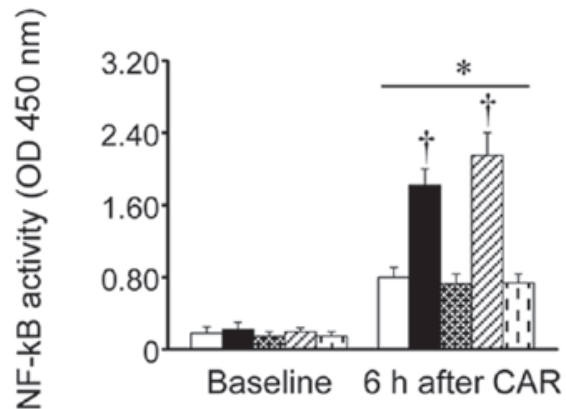

B

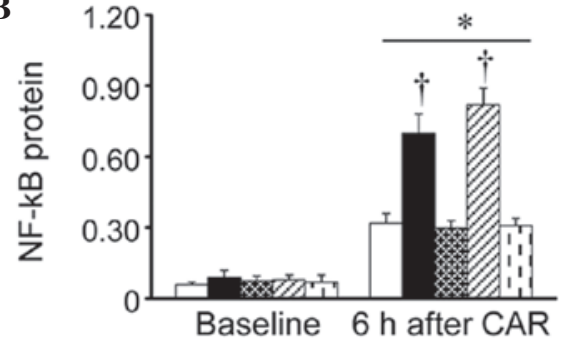

D

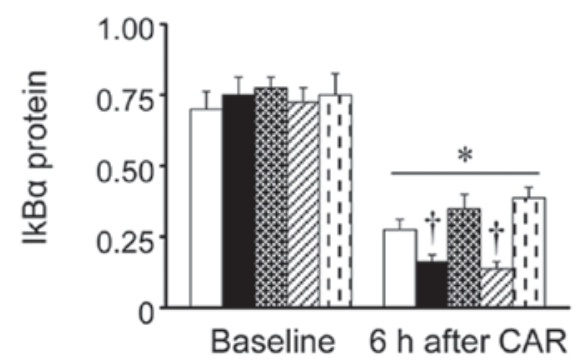

$\square$ LFD HFD control HFD+UA $\square \mathrm{HFD+UA+PPAR} \alpha$ siRNA DHFD+UA+Sc siRNA

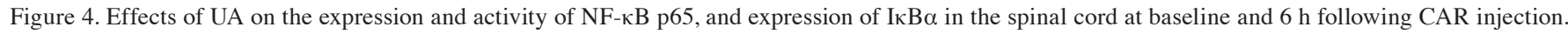
(A) Representative western blots from each group are shown. The (B) expression and activity (C) of NF-kB p65, and (D) expression of IкB $\alpha$ were quantified and corrected by $\beta$-actin. Data are presented as the mean \pm standard error of the mean. ${ }^{*} \mathrm{P}<0.05$, vs. respective baseline; ${ }^{\dagger} \mathrm{P}<0.05$, vs. LFD under the same condition.

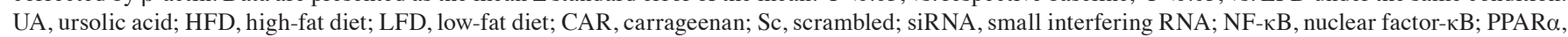

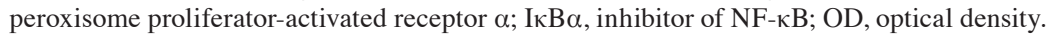

the HFD control rats exhibited increased paw edema between 2 and 24 h following carrageenan injection (Fig. 2C). The HFD+UA rats exhibited significantly reduced paw edema, compared with the HFD control rats. The improvement in paw edema observed in the HFD+UA rats was completely abrogated by the intrathecal injection of PPAR $\alpha$ siRNA, but not scrambled siRNA.
Effects of UA on the inflammatory response in the spinal cord. As the inflammatory response in the spinal cord has been shown to be associated with peripheral inflammation and pain sensitivity, the present study examined the effect of systemic administration of UA on the expression levels of inflammatory mediators and activation of NF-kB, a key mediator of inflammation, in the spinal cord at baseline and $6 \mathrm{~h}$ post-carrageenan injection. The 
A
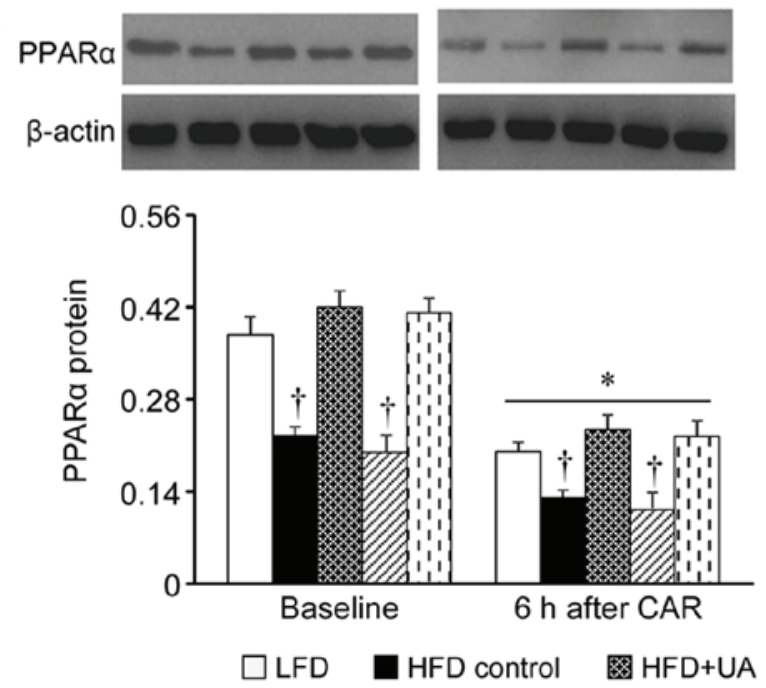

B

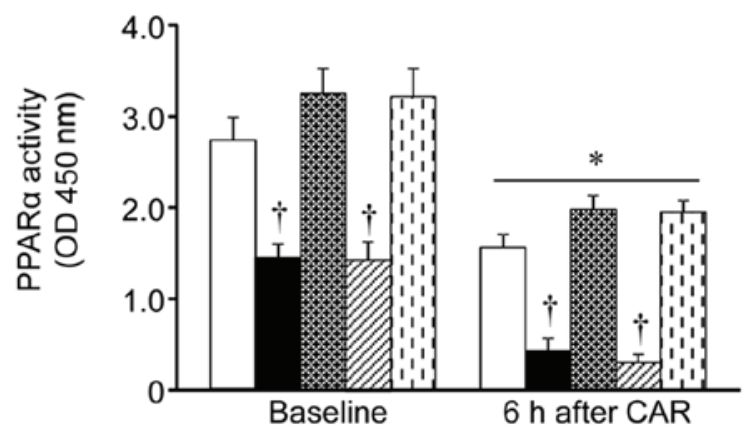

$\square$ HFD+UA+PPAR $\alpha$ siRNA $\square H F D+U A+S c$ siRNA

Figure 5. Effects of UA on PPAR $\alpha$. The (A) expression levels and (B) activity of PPAR $\alpha$ in the spinal cord were determined at baseline and 6 h following CAR injection. Western blot data were corrected by $\beta$-actin. Data are presented as the mean \pm standard error of the mean. * $\mathrm{P}<0.05$, vs. respective baseline; ${ }^{\dagger} \mathrm{P}<0.05$, vs. LFD under the same condition. UA, ursolic acid; HFD, high-fat diet; LFD, low-fat diet; CAR, carrageenan; Sc, scrambled; siRNA, small interfering RNA; PPAR $\alpha$, peroxisome proliferator-activated receptor $\alpha$; OD, optical density.

time points selected were based on previous studies showing that the acute phase of the inflammatory response and hyperalgesia $(0-6 \mathrm{~h})$ is characterized by central sensitization, mediated predominantly by prostanoids, the products of COX-2 $(11,23)$.

No differences were observed in the expression levels of the IL-1 $\beta$, TNF- $\alpha$, COX-2 or iNOS inflammatory mediators (Fig. 3), the activity of NF- $\kappa \mathrm{B}$ p65 or the expression of $\mathrm{I} \kappa \mathrm{B} \alpha$ (Fig. 4) in the spinal cord between the experimental groups at baseline. At $6 \mathrm{~h}$ post-carrageenan injection, all groups of rats exhibited markedly increased expression levels of inflammatory mediators, augmented expression and activity of $\mathrm{NF}-\kappa \mathrm{B}$ p 65, and reduced expression of $\mathrm{I} \kappa \mathrm{B} \alpha$ in the spinal cord, compared with respective baseline values. Notably, the HFD control rats exhibited a more pronounced inflammatory response, which was prevented in the HFD+UA rats. The decreased inflammatory response in the spinal cord of the HFD+UA rats was reversed by intrathecal injection of PPAR $\alpha$ siRNA, but not scrambled siRNA.

Effects of UA on the expression and activity of PPAR $\alpha$ in the spinal cord. To further investigate the molecular mechanisms by which UA attenuates exaggerated inflammatory response in the spinal cord, and prevents augmented peripheral inflammation and inflammatory hyperalgesia in obesity, the present study measured the expression and activity of PPAR $\alpha$ in the spinal cord.

At 12 weeks, the HFD control rats exhibited significant decreases in the expression and activity of PPAR $\alpha$ at baseline, compared with the LFD rats (Fig. 5). Of note, the decreases in the spinal expression and activity of PPAR $\alpha$ were normalized in the HFD+UA rats. However, intrathecal injection of PPAR $\alpha$ siRNA in the HFD+UA rats reduced the spinal expression and activity of PPAR $\alpha$ to the same extent as in the HFD control rats. Intrathecal injection of scrambled siRNA in the HFD+UA rats had no effects on the expression and activity of PPAR $\alpha$.

Carrageenan injection caused significant reductions in the spinal expression and activity of PPAR $\alpha$ in all groups, compared with their baseline values. Of note, the spinal expression and activity of PPAR $\alpha$ remained significantly higher in the LFD rats, HFD+UA rats and HFD+UA rats treated with scrambled siRNA, compared with the other two groups.

\section{Discussion}

The present study reported the novel finding that the systemic administration of UA restored the HFD-induced downregulation of spinal PPAR $\alpha$, which resulted in inhibition of the exaggerated expression of inflammatory mediators in the spinal cord in response to peripheral inflammatory challenge, and the prevention of augmented peripheral pain sensitivity and paw edema in the HFD-induced obese rats. To the best of our knowledge, the present study is the first to report that UA exerted beneficial effects on peripheral inflammation and inflammatory hyperalgesia in obesity via the activation of PPAR $\alpha$ in the spinal cord.

Inflammatory mediators in the CNS have been implicated in the pathogenesis of peripheral inflammation and inflammatory hyperalgesia (10). Peripheral injury induces the increased expression of central inflammatory mediators, particularly COX-2 and iNOS, which are involved in inflammatory signaling to the CNS $(11,24,25)$. Increased expression of COX-2 in the CNS promotes the synthesis and release of prostaglandin E2, contributing to the severity of the inflammatory and peripheral pain responses (24). Carrageenan injection in the paws of rodents can rapidly induce the expression of the proinflammatory cytokines, COX-2 and iNOS, in the spinal cord and other regions of the CNS (24). Emerging evidence suggests that the expression levels of inflammatory mediators in the CNS in response to peripheral inflammatory challenge can be mediated by PPAR $\alpha$, a ligand-activated transcription factor which belongs to the nuclear receptor superfamily (26) and is expressed in the CNS, including the brain and spinal cord $(11,23,27)$. PPAR $\alpha$ is important in regulating lipid metabolism and adipocyte differentiation $(28,29)$. In addi- 
tion, PPAR $\alpha$ is crucial in the regulation of inflammatory responses $(30,31)$. Previous experimental investigation has demonstrated that HFD-induced obesity causes decreases in the expression and activity of PPARy in the spinal cord, which results in the exaggerated expression of inflammatory mediators in response to carrageenan injection in the paw, contributing to augmented peripheral inflammation and inflammatory hyperalgesia (12). UA, a natural pentacyclic triterpenoid and a major bioactive compound in several medicinal herbs, has been shown to increase the expression of PPAR $\alpha$ in the liver in obese animals (14). UA also crosses the blood brain barrier $(20,21)$. In the present study, it was found that the systemic administration of UA for 8 weeks, commencing following a 4-week-period of feeding with a HFD, significantly prevented the carrageenan-induced augmentation of peripheral inflammation and inflammatory hyperalgesia in obese rats, as evidenced by reduced paw edema and increased thermal latency. Molecular investigations revealed that UA restored obesity-induced reductions in the baseline expression and activity of PPAR $\alpha$ in the spinal cord, which led to decreased expression levels of inflammatory mediators and the activity of NF-kB in response to peripheral carrageenan injection. Notably, the expression levels of inflammatory mediators in the spinal cord at baseline were similar among the groups, although the baseline expression and activity of PPAR $\alpha$ were decreased in the HFD control rats, and were normalized in the HFD+UA rats. Following carrageenan injection, the expression levels of inflammatory mediators in the spinal cord were significantly higher in the HFD control rats, compared with the LFD rats, but were attenuated in the HFD+UA rats. These observations suggested that changes in the spinal expression and activity of PPAR $\alpha$ may not affect the expression of inflammatory mediators under normal conditions. However, the decreased spinal expression and activity of PPAR $\alpha$ at baseline may increase susceptibility to peripheral inflammatory stimulation, causing an exaggerated inflammatory response in the spinal cord.

In the present study, it was observed that the abnormal metabolic parameters in obese rats were ameliorated by the systemic administration of UA. These results are consistent with findings of others that UA improves metabolic disorders in HFD-induced obese animals $(13,14)$. However, the restoration of spinal PPAR $\alpha$ by UA may not be entirely attributed to improvement in metabolic disorders, as UA partially improved, but did not normalize, the metabolic parameters in the obese animals. Furthermore, knockdown of spinal PPAR $\alpha$ in the HFD+UA rats by the intrathecal injection of PPAR $\alpha$ siRNA did not alter the UA-induced improvement in metabolic parameters, but reversed the UA-induced decrease in the expression levels of spinal inflammatory mediators, and eliminated the beneficial effects of UA on peripheral inflammation and inflammatory hyperalgesia. These results indicated that the systemic administration of UA inhibited the exaggerated inflammatory responses in the spinal cord in response to peripheral inflammatory stimulation by upregulating spinal $\operatorname{PPAR} \alpha$, thereby preventing augmented peripheral inflammation and inflammatory hyperalgesia.

PPAR $\alpha$ has been suggested to control inflammatory gene expression by modulating the activity of the NF- $\kappa \mathrm{B}$ transcription factor. The expression of a variety of inflammatory genes is driven by $\mathrm{NF}-\kappa \mathrm{B}$, the transcriptional activity of which is regulated by the inhibitory protein, I $\mathrm{B} \alpha$, in the cytoplasm $(32,33)$. Multiple stimuli can rapidly induce the degradation of $\mathrm{I} \kappa \mathrm{B} \alpha$, resulting in translocation of the $\mathrm{NF}-\kappa \mathrm{B}$ complex to the nucleus, where it activates gene transcription (32). The knockout of PPAR $\alpha$ has been shown to increase $N F-\kappa B$ transcriptional activity via downregulation of the cytoplasmic inhibitor, $\mathrm{I} \kappa \mathrm{B} \alpha$ (34), whereas the activation of PPAR $\alpha$ inhibits $\mathrm{NF}-\kappa \mathrm{B}$ transcriptional activity via the upregulation of $\mathrm{I} \kappa \mathrm{B} \alpha(35)$. The results of the present study showed that the systemic administration of UA in obese rats inhibited the augmentation of the expression and activity of $\mathrm{NF}-\kappa \mathrm{B}$ p65 in the spinal cord, which was associated with increased expression of $\mathrm{I} \kappa \mathrm{B} \alpha$ in the cytoplasm. These results suggested that the restoration of spinal PPAR $\alpha$ via systemic administration of UA prevented the exaggerated inflammatory response to peripheral inflammatory stimulation by suppressing spinal $\mathrm{NF}-\kappa \mathrm{B}$ activity. Of note, a previous study demonstrated that the systemic administration of UA attenuates the D-Galactose-induced inflammatory response in the CNS by inhibiting central NF- $\kappa \mathrm{B}$ activity (36).

In conclusion, the present study demonstrated that the systemic administration of UA inhibited the exaggerated spinal cord inflammatory response to peripheral inflammatory stimulation in HFD-induced obesity. This occurred via the restoration of the downregulated expression of spinal PPAR $\alpha$, thereby preventing augmented peripheral inflammation and inflammatory hyperalgesia. These observations suggested that UA may provide a potential therapeutic option for the prevention of increased inflammatory pain in obese patients.

\section{Acknowledgements}

This study was supported by the Youth Science Foundation of Shandong Province, China (grant no. Y2013020046).

\section{References}

1. Prentice AM: The emerging epidemic of obesity in developing countries. Int J Epidemiol 35: 93-99, 2006.

2. Hurt RT, Kulisek C, Buchanan LA and McClave SA: The obesity epidemic: Challenges, health initiatives, and implications for gastroenterologists. Gastroenterol Hepatol (NY) 6: 780-792, 2010.

3. Jankun J, Al-Senaidy A and Skrzypczak-Jankun E: Can inactivators of plasminogen activator inhibitor alleviate the burden of obesity and diabetes? (Review). Int J Mol Med 29: 3-11, 2012.

4. Marcus DA: Obesity and the impact of chronic pain. Clin J Pain 20: 186-191, 2004.

5. Hitt HC, McMillen RC, Thornton-Neaves T, Koch K and Cosby AG: Comorbidity of obesity and pain in a general population: Results from the southern pain prevalence study. J Pain 8: 430-436, 2007.

6. D'Arcy Y: Managing pain in obese patients. Nurse Pract 36: 28-32; quiz 33, 2011

7. Patterson RE, Frank LL, Kristal AR and White E: A comprehensive examination of health conditions associated with obesity in older adults. Am J Prev Med 27: 385-390, 2004.

8. Roane DS and Porter JR: Nociception and opioid-induced analgesia in lean (Fa/-) and obese (fa/fa) Zucker rats. Physiol Behav 38: 215-218, 1986.

9. Croci $T$ and Zarini E: Effect of the cannabinoid CB1 receptor antagonist rimonabant on nociceptive responses and adjuvant-induced arthritis in obese and lean rats. $\mathrm{Br}$ J Pharmacol 150: 559-566, 2007.

10. Iannitti T, Graham A and Dolan S: Increased central and peripheral inflammation and inflammatory hyperalgesia in Zucker rat model of leptin receptor deficiency and genetic obesity. Exp Physiol 97: 1236-1245, 2012. 
11. D'Agostino G, La Rana G, Russo R, Sasso O, Iacono A, Esposito E, Raso GM, Cuzzocrea S, Lo Verme J, Piomelli D, et al: Acute intracerebroventricular administration of palmitoylethanolamide, an endogenous peroxisome proliferator-activated receptor-alpha agonist, modulates carrageenan-induced paw edema in mice. J Pharmacol Exp Ther 322: 1137-1143, 2007.

12. Wang J,Zhang Q,ZhaoL,LiD,FuZ Zand Liang L: Down-regulation of PPAR $\alpha$ in the spinal cord contributes to augmented peripheral inflammation and inflammatory hyperalgesia in diet-induced obese rats. Neuroscience 278: 165-178, 2014.

13. Jia Y, Bhuiyan MJ, Jun HJ, Lee JH, Hoang MH, Lee HJ, Kim N, Lee D, Hwang KY, Hwang BY, et al: Ursolic acid is a PPAR-o agonist that regulates hepatic lipid metabolism. Bioorg Med Chem Lett 21: 5876-5880, 2011.

14. Li S, Liao X, Meng F, Wang Y, Sun Z, Guo F, Li X, Meng M, $\mathrm{Li} \mathrm{Y}$ and Sun C: Therapeutic role of ursolic acid on ameliorating hepatic steatosis and improving metabolic disorders in high-fat diet-induced non-alcoholic fatty liver disease rats. PLoS One 9: e86724, 2014.

15. Liu J: Pharmacology of oleanolic acid and ursolic acid. J Ethnopharmacol 49: 57-68, 1995.

16. Ikeda Y, Murakami A and Ohigashi H: Ursolic acid: An anti- and pro-inflammatory triterpenoid. Mol Nutr Food Res 52: 26-42, 2008.

17. Kim M, Sung B, Kang YJ, Kim DH, Lee Y, Hwang SY, Yoon JH, Yoo MA, Kim CM, Chung HY and Kim ND: The combination of ursolic acid and leucine potentiates the differentiation of $\mathrm{C} 2 \mathrm{C} 12$ murine myoblasts through the mTOR signaling pathway. Int J Mol Med 35: 755-762, 2015

18. Vasconcelos MA, Royo VA, Ferreira DS, Crotti AE, Andrade e Silva ML, Carvalho JC, Bastos JK and Cunha WR: In vivo analgesic and anti-inflammatory activities of ursolic acid and oleanoic acid from Miconia albicans (Melastomataceae). Z Naturforsch C 61: 477-482, 2006.

19. Zhou Y, Li JS, Zhang X, Wu YJ, Huang K and Zheng L: Ursolic acid inhibits early lesions of diabetic nephropathy. Int J Mol Med 26: 565-570, 2010.

20. Tsai SJ and Yin MC: Antioxidative and anti-inflammatory protection of oleanolic acid and ursolic acid in PC12 cells. J Food Sci 73: H174-H178, 2008.

21. Martin R, Carvalho-Tavares J, Hernández M, Arnés M, Ruiz-Gutierrez V and Nieto ML: Beneficial actions of oleanolic acid in an experimental model of multiple sclerosis: A potential therapeutic role. Biochem Pharmacol 79: 198-208, 2010.

22. Madsen AN, Hansen G, Paulsen SJ, Lykkegaard K, Tang-Christensen M, Hansen HS, Levin BE, Larsen PJ, Knudsen LB, Fosgerau K and Vrang N: Long-term characterization of the diet-induced obese and diet-resistant rat model: A polygenetic rat model mimicking the human obesity syndrome. J Endocrinol 206: 287-296, 2010.
23. D'Agostino G, La Rana G, Russo R, Sasso O, Iacono A, Esposito E, Mattace Raso G, Cuzzocrea S, Loverme J, Piomelli D, et al: Central administration of palmitoylethanolamide reduces hyperalgesia in mice via inhibition of NF-kappaB nuclear signalling in dorsal root ganglia. Eur J Pharmacol 613: 54-59, 2009.

24. Ichitani Y, Shi T, Haeggstrom JZ, Samuelsson B and Hökfelt T: Increased levels of cyclooxygenase-2 mRNA in the rat spinal cord after peripheral inflammation: An in situ hybridization study. Neuroreport 8: 2949-2952, 1997.

25. Samad T and Abdi S: Cyclooxygenase- 2 and antagonists in pain management. Curr Opin Anaesthesiol 14: 527-532, 2001.

26. Kota BP, Huang TH and Roufogalis BD: An overview on biological mechanisms of PPARs. Pharmacol Res 51: 85-94, 2005.

27. Moreno S, Farioli-Vecchioli S and Cerù MP: Immunolocalization of peroxisome proliferator-activated receptors and retinoid $\mathrm{X}$ receptors in the adult rat CNS. Neuroscience 123: 131-145, 2004.

28. Berger J and Moller DE: The mechanisms of action of PPARs. Annu Rev Med 53: 409-435, 2002

29. Filip-Ciubotaru F, Foia L, Manciuc C and Grigore C: PPARs: Structure, mechanisms of action and control. Note I. Rev Med Chir Soc Med Nat Iasi 115: 477-484, 2011 (In Romanian).

30. Duval C, Fruchart JC and Staels B: PPAR alpha, fibrates, lipid metabolism and inflammation. Arch Mal Coeur Vaiss 97: 665-672, 2004

31. Cuzzocrea S, Mazzon E, Di Paola R, Peli A, Bonato A, Britti D, Genovese T, Muià C, Crisafulli $\mathrm{C}$ and Caputi AP: The role of the peroxisome proliferator-activated receptor-alpha (PPAR-alpha) in the regulation of acute inflammation. J Leukoc Biol 79: 999-1010, 2006.

32. Auphan N, DiDonato JA, Rosette C, Helmberg A and Karin M: Immunosuppression by glucocorticoids: Inhibition of NF-kappa B activity through induction of I kappa B synthesis. Science 270: 286-290, 1995.

33. George A, Marziniak M, Schäfers M, Toyka KV and Sommer C: Thalidomide treatment in chronic constrictive neuropathy decreases endoneurial tumor necrosis factor-alpha, increases interleukin-10 and has long-term effects on spinal cord dorsal horn met-enkephalin. Pain 88: 267-275, 2000.

34. Zhao W, Iskandar S, Kooshki M, Sharpe JG, Payne V and Robbins ME: Knocking out peroxisome proliferator-activated receptor (PPAR) alpha inhibits radiation-induced apoptosis in the mouse kidney through activation of NF-kappaB and increased expression of IAPs. Radiat Res 167: 581-591, 2007.

35. Vanden Berghe W, Vermeulen L, Delerive P, De Bosscher K, Staels B and Haegeman G: A paradigm for gene regulation: Inflammation, NF-kappaB and PPAR. Adv Exp Med Biol 544: 181-196, 2003.

36. Lu J, Wu DM, Zheng YL, Hu B, Zhang ZF, Ye Q, Liu CM, Shan Q and Wang YJ: Ursolic acid attenuates D-galactose-induced inflammatory response in mouse prefrontal cortex through inhibiting AGEs/RAGE/NF- $\mathrm{B}$ pathway activation. Cereb Cortex 20: 2540-2548, 2010. 